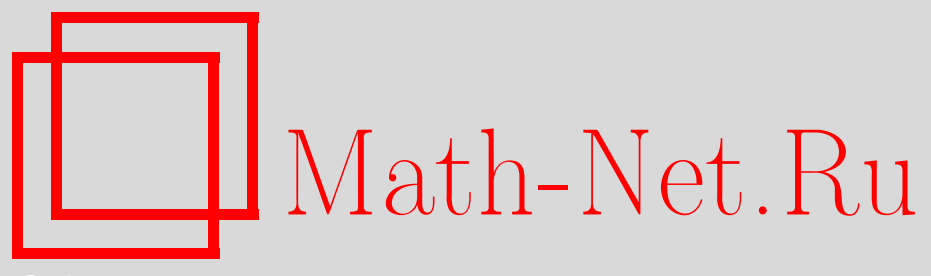

М. Б. Сихов, Н. Т. Темиргалиев, Об алгоритме построения равномерно распределенных сеток Коробова, $M a-$ тем. заметки, 2010, том 87, выпуск 6, 948-950

DOI: https://doi.org/10.4213/mzm7827

Использование Общероссийского математического портала Math-Net.Ru подразумевает, что вы прочитали и согласны с пользовательским соглашением http://www . mathnet.ru/rus/agreement

Параметры загрузки:

IP : 34.229 .108 .108

26 апреля 2023 г., 05:29:12

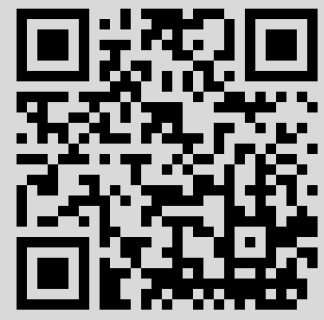




\section{Об алгоритме построения равномерно распределенных сеток Коробова}

\section{М. Б. Сихов, Н. Т. Темиргалиев}

Задача эффективного построения равномерно распределенных сеток на многомерном единичном кубе представляет собой активно развивающееся и перспективное направление с многочисленными приложениями и связями с другими разделами математики (см., например, [1] и имеющуюся в ней библиографию).

В данной статье под равномерной распределенностъю последовательности сеток (конечных множеств) $\left\{\xi_{k}^{(p)}\right\}_{k=1}^{p}$ из $s$-мерного единичного куба $[0,1]^{s}$, индексированных достаточно плотной возрастающей последовательностью целых положительных $p$, понимается существование положительных $c(s)>0$ и $\beta(s)>0$ таких, что для всех $p$ выполнено неравенство

$$
\begin{aligned}
D_{s}\left(\left\{\xi_{k}^{(p)}\right\}_{k=1}^{p}\right) & =\sup \left\{\left|\frac{1}{p} \sum_{k=1}^{p} \chi_{I}\left(\xi_{k}^{(p)}\right)-\prod_{j=1}^{s}\left(d_{j}-b_{j}\right)\right|: I=\prod_{j=1}^{s}\left[b_{j}, d_{j}\right] \subset[0,1]^{s}\right\} \\
& \leqslant c(s) p^{-1}(\ln p)^{\beta(s)}
\end{aligned}
$$

где $\chi_{A}$ - характеристическая функция множества $A$ (подробности см., например, в [2]).

В 1958 г. Коробов [3] установил существование для всякого целого положительного $p$ взаимно простых с $p$ целых чисел

$$
a_{1}=a_{1}(p), \quad a_{2}=a_{2}(p), \quad \ldots, \quad a_{s}=a_{s}(p)
$$

таких, что сетка $(\{x\}-$ дробная часть числа $x)$

$$
\xi_{k}\left(p, a_{1}, \ldots, a_{s}\right)=\left(\left\{\frac{k}{p} a_{1}\right\}, \ldots,\left\{\frac{k}{p} a_{s}\right\}\right), \quad k=1, \ldots, p,
$$

равномерно распределена на $[0,1]^{s}$.

Этот результат является весьма ценным с вычислительной точки зрения в том смысле, что сетка (2) полностью определяется заданием $(s+1)$-мерного целочисленного вектора $\left(p, a_{1}(p), a_{2}(p), \ldots, a_{s}(p)\right)$, по которому она выписывается за $\asymp p$ элементарных арифметических операций, в то время $s$-мерная сетка объема $p$ составляется из $s p$ действительных чисел.

Сетки вида (2) назовем сетками Коробова.

Таким образом, задача заключается в нахождении достаточно плотной последовательности целых положительных $p$ и соответствующих им взаимно простых с $p$ целых чисел $a_{1}(p), a_{2}(p), \ldots, a_{s}(p)$ таких, что для дискрепанса $D_{s}$ сетки Коробова (2) выполнено неравенство (1).

Основным результатом настоящей работы является следующая теорема, в которой дан определенный ответ на поставленную задачу. Формулировке этой теоремы предпошлем еще одно определение. Положим

$$
b_{r}(x)=\sum_{\left(m_{1}, \ldots, m_{s}\right) \in \mathbb{Z}^{s}}\left(\bar{m}_{1} \cdots \bar{m}_{s}\right)^{-r} e^{2 \pi i\left(m_{1} x_{1}+\cdots+m_{s} x_{s}\right)},
$$

где $r>1$ и $\bar{m}_{j}=\max \left(1,\left|m_{j}\right|\right), j=1, \ldots, s$. 
Теорема. При данных $r>1 u s, s=1,2, \ldots$, существуют положительные величины $c_{1}, c_{2}, c_{3}, \beta_{1}$ и $\beta_{2}$ такие, что для всякого челого положительного $p$ и для всякого целочисленного вектора $\left(a_{1}, \ldots, a_{s}\right)$ неравенство

$$
c_{3}(s) \frac{(\ln p)^{(s-1) / 2}}{p} \leqslant D_{s}\left[\left(\left\{\frac{k}{p} a_{1}\right\}, \ldots,\left\{\frac{k}{p} a_{s}\right\}\right)_{k=1}^{p}\right] \leqslant c_{1}(s) \frac{(\ln p)^{\beta_{1}(s)}}{p}
$$

выполнено тогда и только тогда, когда

$$
\left|\frac{1}{p} \sum_{k=1}^{p} b_{r}\left(\left\{\frac{k}{p} a_{1}\right\}, \ldots,\left\{\frac{k}{p} a_{s}\right\}\right)-1\right| \leqslant c_{2}(r, s) \frac{(\ln p)^{\beta_{2}(r, s)}}{p^{r}} .
$$

Оценка снизу в (3) следует из результатов Рота [1].

В этой теореме в дополнение к известным (см. в [3] теорему 22 (стр. 141-146) и теорему 19 (стр. 126-130)) дан еще один критерий равномерной распределенности сеток Коробова.

Задаче построения оптимальных квадратурных формул, эквивалентной выполнению неравенства (3) (подробности см. в [3]), посвящена обширная литература (см., например, [2]-[4] и имеющуюся в них библиографию); результаты этих работ носят в основном характер теорем существования.

В [5]-[7] даны эффективные алгоритмы построения равномерно распределенных сеток [7; теорема 2] и оптимальных в степенной шкале квадратурных формул с равными весами и сеткой вида (2) [7; теорема 4], сводящиеся к делению целых рациональных чисел.

Все эти алгоритмы, даже при своей оптимальности относительно числа элементарных арифметических операций $~ N \ln ^{\tau} N, \tau \geqslant 0$, при достаточном числе узлов $N$ для реализации требуют большой вычислительной работы.

Поэтому для построения конкретных равномерно распределенных сеток приходится привлекать другие методы. $\mathrm{K}$ таковым относятся вычислительные эксперименты, основанные на приведенной выше теореме.

Именно, в случае целых $r>1$ функция $b_{r}(x)$ из условия (4) есть 1-периодический алгебраический многочлен Бернулли, например, при $r=10$

$$
b_{r}(x)=\prod_{j=1}^{s}\left[1+\frac{(2 \pi)^{10}}{10 !}\left(\frac{5}{66}-\frac{3}{2} x_{j}^{2}+5 x_{j}^{4}-7 x_{j}^{6}+\frac{15}{2} x_{j}^{8}-5 x_{j}^{9}+x_{j}^{10}\right)\right] .
$$

Более того, поскольку в (3) параметр $r>1$ участвует только в константах, то тем самым, для доказательства неравенства (4) для всех $r>1$ достаточно это неравенство доказать для некоторого $r=r_{0}>1$ (с точностью до констант).

Таким образом, задача построения равномерно распределенной сетки Коробова сведена к проверке выполнения неравенства (4) при каком-либо целом $r>1$ для алгебраического многочлена Бернулли $b_{r}(x)$ (одно из реализаций которого дано в [8]).

\section{СПИСОК ЦИТИРОВАННОЙ ЛИТЕРАТУРЫ}

[1] K.F. Roth, Математика: границы и перспективы, ФАЗИС, М., 2005, 375-394. [2] Л. Кейперс, Г. Нидеррейтер, Равномерное распределение последователъностей, Наука, М., 1985. [3] Н. М. Коробов, Теоретико-числовые методы в приближенном анализе, Физматгиз, М., 1963. [4] L. K. Hua, Y. Wang, Applications of Number Theory to Numerical Analysis, Springer-Verlag, Berlin, 1981. [5] С. М. Воронин, Н. Темиргалиев, Матем. заметки, 46:2 (1989), 34-41. [6] С. М. Воронин, Избранные труды. Математика, Изд-во 
МГТУ им. Н.Э. Баумана, М., 2006. [7] Н. Темиргалиев, Е. А. Баилов, А. Ж. ЖКубанышева, Докл. РАН, 416:2 (2007), 169-173. [8] А. Ж. Жубанышева, Н. Темиргалиев, Ж. Н. Темиргалиева, ЖВМ и МФ, 49:1 (2009), 14-25.

\section{М. Б. Сихов}

Казахский национальный университет им. аль-Фараби,

Евразийский национальный университет им. Л. Н. Гумилёва

E-mail: mirbulats@mail.ru

\section{Н. Т. Темиргалиев}

Казахский национальный университет им. аль-Фараби,

Евразийский национальный университет им. Л. Н. Гумилёва

E-mail: ntmath29@mail.ru 